\title{
AN IMPROVED DOUBLE CODING LOCAL BINARY PATTERN ALGORITHM FOR FACE RECOGNITION
}

\author{
Saurabh Asija ${ }^{1}$, Rakesh Singh ${ }^{2}$ \\ ${ }^{1}$ Research Scholar (Computer Engineering Department), Punjabi University, Patiala. \\ ${ }^{2}$ Asst. Professor (Computer Engineering Department), Punjabi University, Patiala.
}

\begin{abstract}
A human face conveys a lot of information about the identity and emotional state of the person. So now a day's face recognition has become an interesting and challenging problem. Face recognition plays a vital role in many applications such as authenticating a person, system security, verification and identification for law enforcement and personal identification among others. So our research work mainly consists of three parts, namely face representation, feature extraction and classification. The first part, Face representation represents how to model a face and check which algorithms can be used for detection and recognition purpose. In the second phase i.e. feature extraction phase we compute the unique features of the face image. In the classification phase the computed DLBP face image is compared with the images from the database. In our research work, we use Double Coding Local Binary Patterns to evaluate face recognition which concentrate over both the shape and texture information to represent face images for person independent face recognition. The face area is firstly cut into small regions from which Local Binary Patterns (LBP), then we compute histograms to generate LBP image then we compute single oriented mean image from which we again compute histogram values small regions and at last concatenated into a single feature vectors and generate D-LBP image. This feature are used for the representation of the face and to measure similarities between images.
\end{abstract}

Keywords: local binary pattern (LBP), double coding local binary pattern (D-LBP), features extraction, classification, pattern recognition, histogram, feature vector.

\section{INTRODUCTION}

Image processing can be defined as any form of signal processing which takes an image as a input, which can be a video frame or a photograph for which the output can be an image itself or a set of its characteristics or parameters closely related to the image. Face Recognition is a biometric trait used widely now a days and it make use of computer software to identify of the individual. In face recognition the automatic recognition of a person take place using distinguishing traits. In social life our main focus is on face, which plays a vital role in conveying identity and emotions. We recognize many of faces seen in our life and identify familiar faces at a short stamp even after many years of gap. Because of quite robustness, despite large changes in the visual stimulus due to age, viewing effects, beards, and distractions such as glasses, expression or changes in hair style. Face recognition plays a vital role in many applications such as authenticating a person, system security, verification and identification for law enforcement and personal identification among others aspects [7].

Local binary pattern (LBP) is used in computer vision for the purpose classification. In Texture Spectrum model LBP was proposed in 1990. LBP was first described in 1994. (LBP) is a texture operator simple yet efficient in use. it extracts pixels of an image and label them by calculating the threshold of the neighbors of each pixel and then consider the result in binary form. Due to its computational simplicity and discriminative power, it proves to be a better approach in various applications.

Double coding Local binary patterns is an invariant of LBP. In LBP we compute only amplitude threshold $\theta$ while in $d-$ LBP we compute distance threshold too $\varepsilon$.

$$
\begin{array}{ll}
\theta=1 / p \sum_{k=0}^{p-1}\left|\mathrm{i}_{\mathrm{k}}-\mathrm{i}_{\mathrm{c}}\right|, \mathrm{p} & =4 \\
\varepsilon=1 / p \sum_{k=0}^{p-1} \mathrm{i}_{\mathrm{k}}-\mathrm{i}_{\mathrm{c}}, \mathrm{p} & =4
\end{array}
$$

\section{FACE RECOGNITION PROCESS}

Although there are number of professional and commercial face recognition systems yet this way of identification continues to be an interesting topic for researchers. This is due to the fact that the under the feasible conditions the current systems perform well but when variations occurs its performance decreases. The variation factors are viewpoint, facial expressions, time (when the pictures are made), pose and illumination (lightening changes)[8]. The goal in this research area is to minimize the influence of these factors and create robust face recognition system. A model for face recognition is shown in Figure-1.1. 


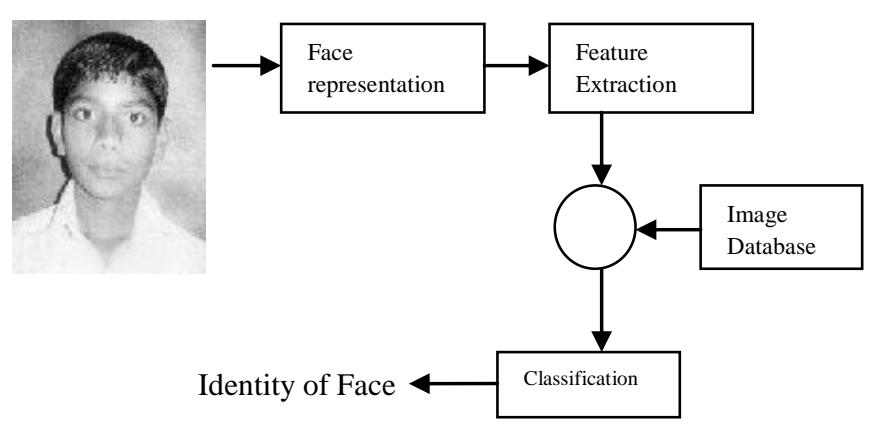

Figure 1.1: Principle of an identification process with Face Recognition

The process of person identification by using face recognition can be split into three main phases (figure 1.1). These are face representation, feature extraction and classification [3].The first part, Face representation represents how to model a face and check which algorithms can be used for detection and recognition purpose. In the second phase i.e. feature extraction phase we compute the unique features of the face image. In the classification phase the computed DLBP face image is compared with the images from the database [3]. The output of the classification part is the identity of a face image from the database with the highest matching score, thus with the smallest differences compared to the input face image. Also a threshold value can be used to determine if the differences are small enough. After all, it could be that a certain face is not in the database at all.

\section{LOCAL BINARY PATTERN}

For face recognition there exist several methods to extract the most useful features from the face images .Now a day's Local Binary Pattern (LBP) method is mostly used method for feature extraction. This relative new approach was introduced in 1996 by Ojala et al. [3]. With the help of LBP it become easy to describe the shape and texture of a face image. In this method for the purpose of feature extraction an image is divided into several small regions. From each individual region the features are extracted.

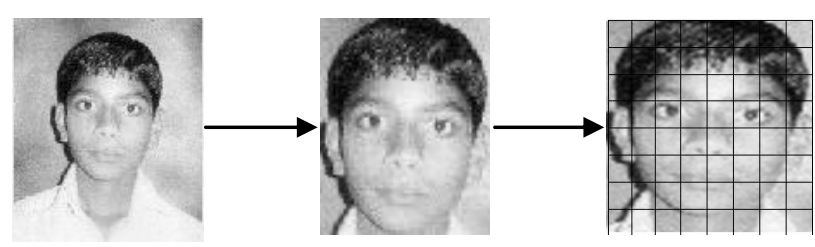

Figure 1.2: A preprocessed image divided into 64 regions

These features are coded into binary patterns to describe the surroundings of pixels in the regions. Each and every region is processed to compute the features and these computed features from all regions are combined into a single feature histogram, which forms a representation of the image. Images are compared by measuring the similarity between their histograms. According to studies face recognition using the LBP method had provided very good results, both in terms of speed and discrimination performance. Due to its describing power of image texture and shape it seems to be quite useful in recognizing face images with different lightening conditions, image rotation, different facial expressions and aging of persons.

\section{DOUBLECODINGLOCAL BINARY PATTERN}

Basic LBP operator works on center pixel and neighborhood pixels. It computes the differences between the gray values between center pixel and neighborhood pixels, but the amplitude relationship between center pixel gray value and the neighborhood pixel gray values are ignored. Because every pixel gray value cannot be made full use of, it could lead to a drop in the final recognition rate when the face texture features are extracted. Moreover, too much sampling points will make the algorithm more complicated, which may result in the decrease of the rate of recognition. Due to the this problems, this we presents a double coding local binary pattern (d-LBP). Firstly, to reduce the complexity of the algorithm, the sampling points of d-LBP operator is reduced to 4 from 8 of the basic LBP operator
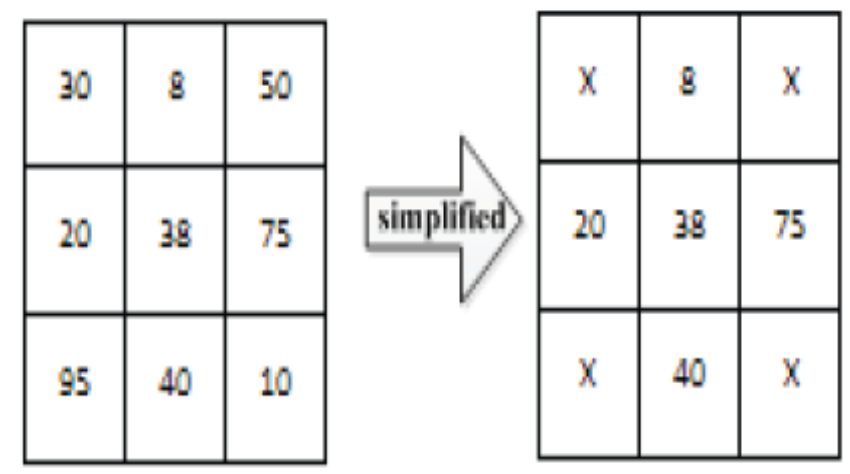

Figure 1.3: Reducing Sample Size

Then, making full use of the relationship among the gray values of each pixel within a certain local area. So $\theta$ is defined as the amplitude threshold and $\Sigma$ is defined as the difference threshold [7].

$$
\begin{gathered}
\theta=1 / p \sum_{k=0}^{p-1}\left|\mathrm{i}_{\mathrm{k}}-\mathrm{i}_{\mathrm{c}}\right|, \mathrm{p}=4 \\
\varepsilon=1 / p \sum_{k=0}^{p-1} \mathrm{i}_{\mathrm{k}}-\mathrm{i}_{\mathrm{c}} \quad, \mathrm{p}=4
\end{gathered}
$$

Where: $i_{c}$ is the gray value of the center pixel in the local area; $i_{k}$ shows the $\mathrm{k}$-th grey value of sampling point in the center pixel neighborhood area; $p$ is the number of sampling points. Finally, in order to describe the facial texture information in detail, the basic LBP operator with one binary coding is replaced by the improved LBP operator with two binary encoding. The first binary code is related with the difference in gray value of the neighborhood pixels and the center pixel. Compared with difference threshold $(\Sigma)$ if it is larger, the binary code is 1 , on the other hand, is marked as 0 . The second binary code is related with amplitude between neighborhood pixels gray values and the center pixel gray value. Compared with amplitude threshold $(\theta)$, if it is larger, the binary code is marked as 1 , otherwise 0 . As shown in formula [7]. 
$\mathrm{dLBP}=\sum_{k}^{p-1} 2^{2 k} s\left(i_{k}, i_{c}\right)$

$s\left(i_{k}, i_{c}\right)=\left\{\begin{array}{l}00, i_{k}-i_{c}<\varepsilon \equiv\left|i_{k}-i_{c}\right|<\theta \\ 01, i_{k}-i_{c}<\varepsilon \equiv\left|i_{k}-i_{c}\right|>\theta \\ 10, i_{k}-i_{c}>\varepsilon \equiv\left|i_{k}-i_{c}\right|<\theta \\ 11, i_{k}-i_{c}>\varepsilon \equiv\left|i_{k}-i_{c}\right|>\theta\end{array}\right.$

$\mathrm{p}=4$

\begin{tabular}{|c|c|c|}
\hline$x$ & 8 & $x$ \\
\hline 20 & 38 & 75 \\
\hline$x$ & 40 & $x$ \\
\hline
\end{tabular}

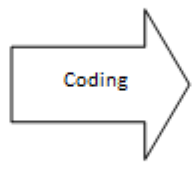

\begin{tabular}{|c|c|c|}
\hline$x$ & 01 & $x$ \\
\hline 00 & 38 & 10 \\
\hline$x$ & 11 & $x$ \\
\hline
\end{tabular}

Figure 1.4: Double Binary Coding

$\theta=1 / 4(0+37+2+18)=22$;

$\varepsilon=1 / 4(8+75+40+20)-38=-2$;

$\mathrm{d}-\mathrm{LBP}=01111000=120$

In order to increase the accuracy of the d-LBP operator and make better use of d-LBP to describe face image. First of all, the d-LBP map of face image should be blocked; then, the images of each block are calculated to get the histogram of d-LBP respectively; finally, each partitioned histograms is connected according to a certain order to get a composite feature vector, that is, the d-LBP histogram of overall face image.

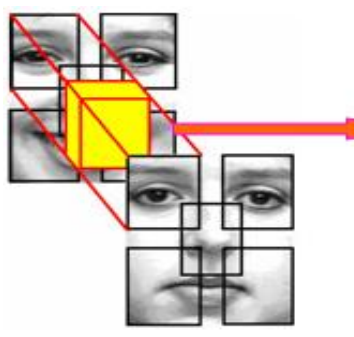

(a)

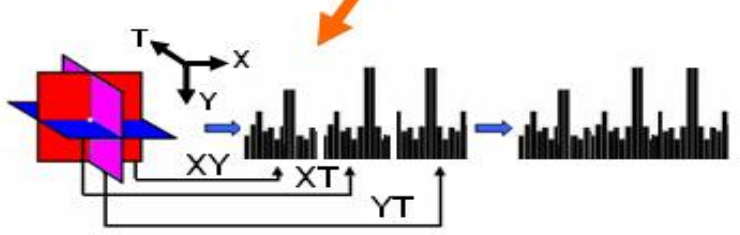

(c)
Nonparametric statistical method is used to determine the histogram similarity between samples after getting a d-LBP histogram.

$$
\varphi^{2}\left(H_{1}, H_{2}\right)=\sum_{i} \frac{\left(\mathrm{H}_{1}(\mathrm{i})-\mathrm{H}_{2}(\mathrm{i})\right)^{2}}{\mathrm{H}_{1}(\mathrm{i})+\mathrm{H}_{2}(\mathrm{i})}
$$

Where: is the training sample; $H_{1}(\mathrm{i})$ is the sample to be classified; Similarity $\mathrm{H}_{2}$ (i) is measured by the distance of $\varphi^{2}$, the smaller the $\mathrm{d}$ istance, the more similar the two faces.

\subsection{Implementation}

The process of Face recognition is not a simple problem because when input image is an unknown then face image seen in the extraction phase is usually different from the face image seen in the classification phase. Although local binary features has been extracted from the face image and DLBP image is computed for face recognition that there are several face image uses in the database that compared with the input face image. The face image depends on viewing lighting and environmental conditions. In addition the face image changes according to the expressions. In the research work, which is flexible and efficient, should be solved the problems.

\subsection{D-Lbp Face Recognition Algorithm}

To implement the face recognition in this research work, we proposed the Double Coding Local Binary patterns methodology. D-LBP works on local features that uses DLBP operator which summarizes the local special structure of a face image.

In order to describe the facial texture information in detail, the basic LBP operator with one binary coding is replaced by the improved LBP operator with two binary encoding. The first binary code is related with the difference in gray value of the neighborhood pixels and the center pixel. Compared with difference threshold $(\Sigma)$, if it is larger, the binary code is 1 , on the other hand, is marked as 0 . The second binary code is related with amplitude between neighborhood pixels gray values and the center pixel gray value. Compared with amplitude threshold $(\theta)$, if it is larger, the binary code is marked as 1 , otherwise 0 .

$$
\begin{aligned}
& \mathrm{dLBP}=\sum_{k}^{p-1} 2^{2 k} s\left(i_{k}, i_{c}\right) \\
& \mathrm{p}=4\left(i_{k}, i_{c}\right)=\left\{\begin{array}{l}
00, i_{k}-i_{c}<\varepsilon \equiv\left|i_{k}-i_{c}\right|<\theta \\
01, i_{k}-i_{c}<\varepsilon \equiv\left|i_{k}-i_{c}\right|>\theta \\
10, i_{k}-i_{c}>\varepsilon \equiv\left|i_{k}-i_{c}\right|<\theta \\
11, i_{k}-i_{c}>\varepsilon \equiv\left|i_{k}-i_{c}\right|>\theta
\end{array}\right.
\end{aligned}
$$

The Face Recognition Algorithm

Input: Training Image set.

Output: Feature extracted from face image and compared with centre pixel and recognition with unknown face image.

1) Initialize temp $1=0$

2) FOR each image $I_{m}$ in the training set)

3) Initialize the LBP histogram, $\mathrm{H}=0$

4) FOR each center pixel $i_{c}$ in $I_{m}$

5) Compute the gray value of $i_{c}, \operatorname{LBP}(1)$

6) Increase the corresponding bit by 1 .

7) END FOR LOOP

8) Compute the LBP feature for each facial image and combined into single vector.

9) Compare it with test image in database.

10) If it matches it's most similar face in database then successfully recognized 


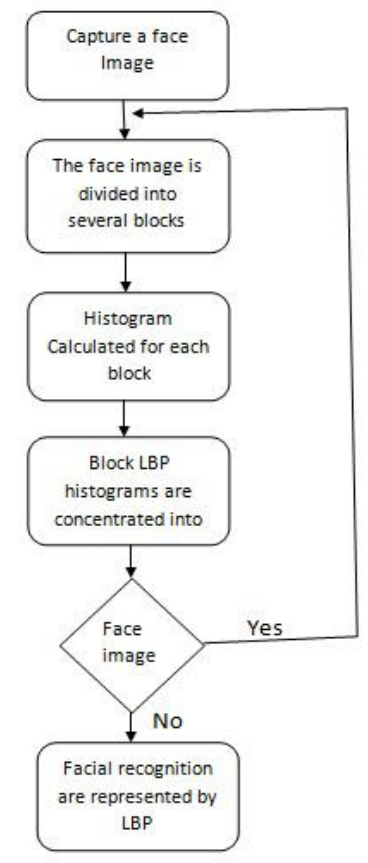

Figure 1.6: Flowchart of the LBP Process

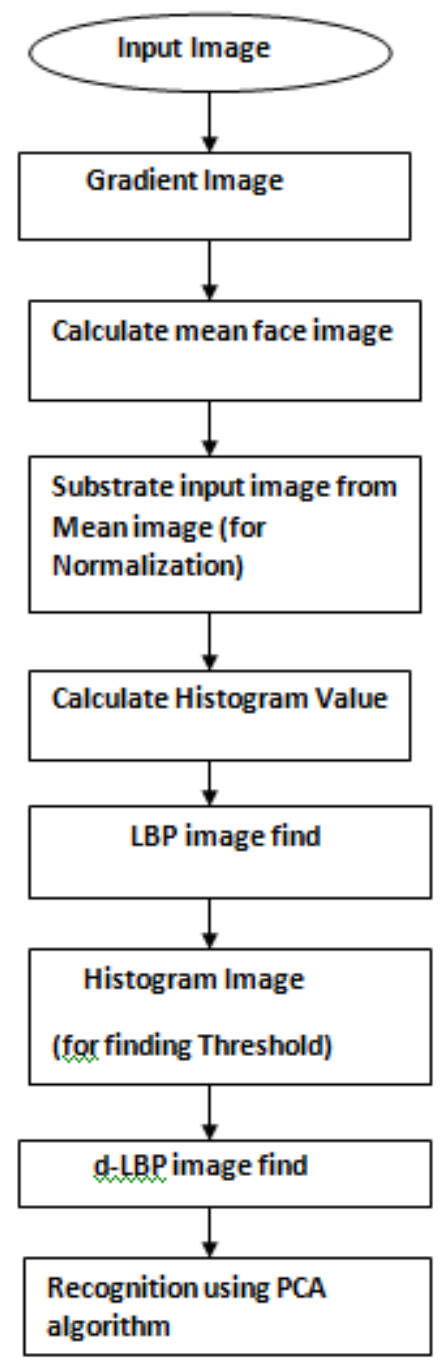

\section{RESULTS AND DISCUSSION}

This performance of the DLBP-method is tested by implementation on different kind of face images. The image is divided into of the 64 regions several parameters like LBP image, DLBP operator, non-weighted or weighted regions are computed and judge the influence of these parameters on the performance. For this research purpose we have collected lots of face images.

We compare the face image of an unknown identity with face images of known individuals from a large database. In the figure 1.8 we can see the input facial images used for input for face recognition are given below:

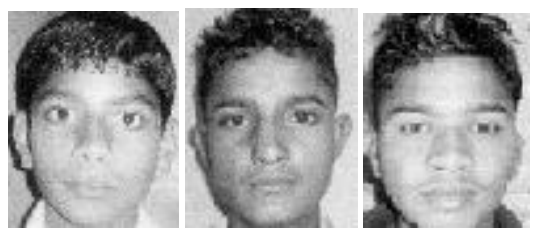

Figure 1.8: Input images

And also in the figure 1.9 we can see the facial images that are stored in the database which compared with the input facial images. If the input face images are found or the more similarities face images are matched in the database then we say the face image is successfully recognized.

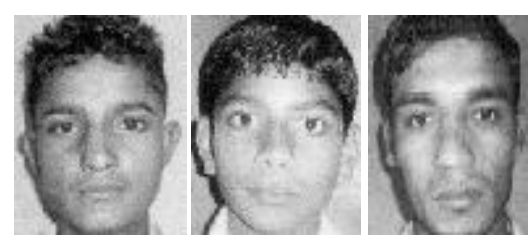

Figure 1.9: Database images

Based on the algorithm the input face images are compared with database facial images for identification. The face recognition results are shown in below in window mode:

Figure 1.7: Flowchart of the Proposed System 


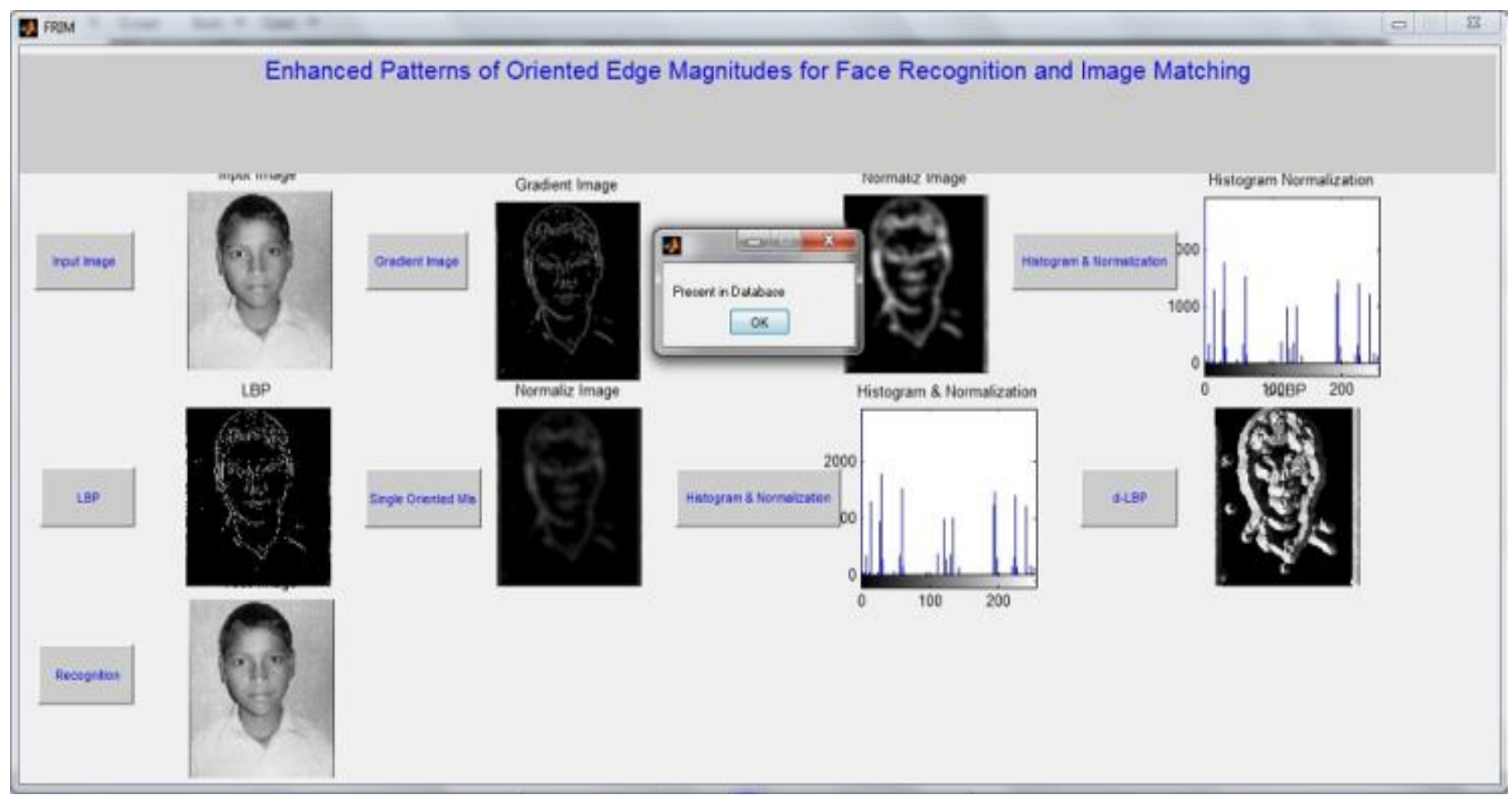

Figure 1.10: Face recognition And image matching window

The following table shows overall unit training time:

\begin{tabular}{|l|l|l|l|l|}
\hline & $\operatorname{LBP}_{(1,4)}$ & $\operatorname{LBP}_{(1,8)}$ & DLBP & $\begin{array}{l}\text { DLBP } \\
\text { using } \\
\text { gradient } \\
\text { image and } \\
\text { SIMs }\end{array}$ \\
\hline $\begin{array}{l}\text { Unit } \\
\text { Training } \\
\text { Time(ms) }\end{array}$ & 3.25 & 3.75 & 3.35 & 3.15 \\
\hline
\end{tabular}

\section{CONCLUSION}

In this research, work has been done to improve the performance of a face recognition system by making use of DLBP for feature extraction. The local feature of the face are extracted and matched with the most similar face image in database. The accuracy of the system is above $100 \%$ by the DLBP algorithm. The result of this face recognition system is good but there is scope for future improvement. The main improvement will pursue the performances, recognizes the real-time face recognition. I would like to improve my code for face image recognition as well as clean up the code in order to improve performance.

Many difficulties has been faced when recognized face images from database such as pose and lighting variations, expression variations, age variations, and facial occlusions. In future to improve the pose correction, quality based frame selection, aging correction, and mark based matching techniques can be combined to build a unified system for video based face recognition.

\section{REFERENCES}

[1]. Aaima Najam, Zeeshan Ahmed and Faizan Ahmad( Image-based Face Detection and Recognition:"State of the Art") International Journal of Computer Science Issues (IJCSI) Volume 9, Issue 6, November 2012
[2]. Zhimin Cao(Face Recognition with Learning-based Descriptor), The Chinese University of Hong Kong

[3]. Md. Abdur Rahim, Md. Najmul Hossain (Face Recognition using Local Binary Patterns (LBP)), Global Journal of Computer Science and Technology Graphics \& Vision Volume 13 Issue 4 Version 1.0 Year 2013 Online ISSN: 0975-4172 \& Print ISSN: 0975-4350

[4]. Alex P. Pentland and Matthew A. Turk (Face Recognition using Eigenfaces), Vision and Modeling Group, The Media Laboratory, Massachusetts Institute of technology

[5]. Hong-Jiang Zhang, Yuxiao Hu, Xiaofei He, , Partha Niyogi, and Shuicheng Yan (Face Recognition Using Laplacianfaces) IEEE Transaction of pattern analysis and machine intelligence, Vol 27,No 3, March 2005

[6]. Naoufel Werghi(The mesh-LBP: Computing Local Binary Patterns on Discrete Manifolds) 2013 IEEE International Conference on Computer Vision Workshops

[7]. Wael Louis, K.N. Plataniotis (Frontal Face Datection For Survellance Purpose Using Dual Local Bianry Patterns Features) Multimedia Lab, Department of Electrical and Computer Engineering, University of Toronto, Canada

[8]. Gao Ye, Gao Kao (The Face Recognition Algorithm Based On Double Coding Local Binary Pattern) School of Computer Science and Technology, Xi`an university of Science and Technology Xi`an, 710054, China, 978-1-4799-4169-8/14 \$31.00 @ 2014 IEEE

[9]. W. Zhao, R. Chellappa, P. J. Phillips, and A. Rosenfeld, "Face recognition: A literature survey" ACM Computing Surveys (CSUR), 35(4):399\{458, 2003. 


\section{BIOGRAPHIES}

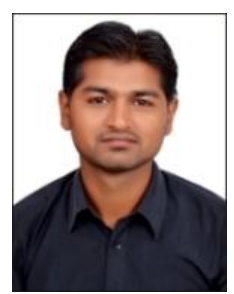

Saurabh Asija He received the B.Tech Degree in computer science from BGIET, Sangrur, in 2011.He is currently working toward the M.Tech degree in the Department of Computer Engineering, Punjabi University, Patiala. His research interests are image processing and computer vision.

Rakesh Singh, He is Asst. Professor, Computer Engineering Department, Punjabi University, Patiala. His area of interest is image processing and computer vision. 